Rheumatoid arthritis (RA) patients are at higher risks of bacterial infection than healthy subjects. Polymorphonuclear leukocytes (PMN) are the first line of nonspecific cellular defence against these infections. We tested the hypothesis that abnormal directed migration of PMN may be one reason for the increased infection rate of RA patients. PMN migration was investigated in 68 peripheral blood samples of 15 RA patients compared with 64 samples of healthy controls in a novel whole blood in vitro membrane filter assay. The migration of PMNs from RA patients and controls was stimulated using the bacterial chem oattractan $\mathrm{N}$-for m yl-m ethionyl-leucylphenylalanine (fMLP). Unstimulated PMN migration of RA patients was increased compared with healthy controls as measured by the following parameters: (a) absolute number of migrant PMNs $(1954 \pm 87$ vs. 1238 $\pm 58 \mathrm{PMN} / \mathrm{m} \mathrm{m}^{2}$ ), (b) percentage of PMNs migrated in to the filter (total $\mathrm{m}$ igration index, TMI) $(28.6 \pm 0.9$ vs. $24.0 \pm 0.8 \%$ ), (c) the distance half the migrating PMNs had covered (distribution characteristic, DC) $(22.6 \pm 1.1 \mathrm{vs} .16 .1 \pm 0.6 \mathrm{~mm})$ and $(\mathrm{d})$ the product of TMI and DC (neutrophil migratory activity, NMA) $(669.0 \pm 45.0$ vs. $389.0 \pm 18.9)$. fMLP stimulated PMNs of RA patients showed defective migration compared to unstimulated samples as shown by (a) a reduced number of migrant PMNs (1799 $\left.\pm 93 \mathrm{PMN} / \mathrm{m} \mathrm{m}^{2}\right)$, (b) lower TMI $(26.1 \pm 0.9 \%)$, (c) unremarkable altered distribution characteristic $(22.9 \pm 0.8 \mathrm{~mm})$ and $(\mathrm{d})$ significant reduced $m$ igratory activity $(600.0 \pm 30.0)$. Our data suggest that the high incidence of infections in RA patients may partly be caused by defective migratory activity of PMNs to bacterial chemoattractants as demonstrated by fMLP.

Key words: Rheumatoid arthritis, Polymorphonuclear leukocytes, Migration, Whole blood

\section{Abnormal directed migration of blood polymorphonuclear leukocytes in rheumatoid arthritis. Potential role in increased susceptibility to bacterial infections}

\author{
F. Aglas, ${ }^{1, C A}$ J. Hermann ${ }^{1}$ and G. Egger ${ }^{2}$
}

${ }^{1}$ Department of Internal Medicine and ${ }^{2}$ Institute for General and Experimental Pathology, Karl Franzens University, Graz, Austria

${ }^{\mathrm{CA}}$ Corresponding Author

Tel: (+43) 3163852274

Fax: $(+43) 3163853062$

\section{Introduction}

A 2-10 times increased susceptibility for bacterial infections ${ }^{1-5}$ has been proposed in patients with rheumatoid arthritis (RA). This has been primarily attributed to immunosuppressive treatments widely used in RA. Other authors believe that RA itself predisposes to infectious diseases. ${ }^{6}$

Polymorphonuclear leukocytes (PMN) belong to the first line of nonspecific cellular defense against microbiological organisms. Their failure can lead to life threatening infections as demonstrated by inherited disorders like Kostmann syndrome. Some studies have shown impaired PMN function in RA patients ${ }^{7-11}$ whereas others have failed to confirm these findings. ${ }^{12-15}$ These controversial studies may be the result of different experimental designs. Chemotax is of PMN has usually been investigated on separated cell preparations. A recent assessment has shown that the separating procedure itself causes a PMN sub-lethal injury, ${ }^{16}$ which may lead to artefacts in the migration assay. Therefore, the activation state of PMNs is influenced to an unforeseeable degree. Whole blood samples have been shown to be advantageous to separated cell preparations in the examination of PMN migration. ${ }^{16,17}$ Fresh whole blood preserves the genuine priming state of PMNs to a high extent. To our knowledge, migration of PMNs in such a whole blood assay has not been investigated thoroughly in RA.

In our ex vivo migration assay $\mathrm{N}$-formyl-me thionylleucyl-phenylalanine (fMLP) a bacterial chemotactic peptid was used in a solid form. This reagent is similar to lipopolysaccharide in stimulating recruitment of PMNs to inflammatory sites and its activity has been demonstrated in different assays. 
We report on spontaneous and chemoattractant directed neutrophil migration using a recently described whole blood membrane filter assay which is a sensitive and diagnostically predictive method to estimate the original priming state of blood PMNs in juvenile RA. ${ }^{17}$

\section{Patients and Methods}

Between one and 13 fresh whole blood samples (a total of 68 samples) were collected from each of 15 patients (14 females, one male; mean age $60.0 \pm$ SEM 10.9 years, range 35-72 years) with RA defined according to the 1987 ARA criteria. Migration tests were performed in different states of disease activity and compared with reactions of 64 healthy controls (25 females, 39 males; mean age $24.0 \pm$ SEM 24.1 years, range 3 weeks to 92 years). In the control group juveniles and adults without any clinical and chemical signs of inflammation were included.

PMN migration was measured in fresh whole blood by a novel membrane filter device as previously described in detail by Egger and coworkers. ${ }^{17}$ The migration chamber consisted of a cellulose nitrate membrane filter of $3 \mathrm{~mm}$ pore size and $140 \mathrm{~mm}$ thickness. In the directed (stimulated) migration experiments a chemoattractant depot containing fMLP was attached. Unstimulated experiments lacked fMLP. A circular plastic container was attached to the membrane filter. Fresh whole blood 1:3 diluted in Hanks's balanced salt solution prewarmed to $37^{\circ} \mathrm{C}$, PMNs migrated from the blood suspension into the membrane filter. PMNs were fixed, stained and counted under the microscope as described elsewhere. ${ }^{17}$ The counting was performed in consecutive steps of $10 \mu \mathrm{m}$ thickness indicating the PMN distribution throughout the whole depth of the filter. Results were evaluated by the following 'migration parameters': (a) absolute number of migrant PMNs (PMN/ $\mathrm{mm}^{2}$ ), (b) percentage of PMNs migrated from the blood total into the filter (total migration index, TM), (c) the distance $(\mathrm{mm})$ half the migrating PMNs had covered within the filter (distribution characteristic, DC) and (d) the product of TMI and DC (neutrophil migratory activity, NMA), expressing the migratory capacity of a PMN collective. Reactions referred to the chemoattractant fMLP (stimulated conditions) and to blanks (unstimulated conditions). Each experiment was performed in triplicate. Results in RA patients were compared with those in healthy controls.

PMN numbers per microlitre of blood and PMN numbers counted in the filters were loaded directly onto a computer. A program calculated and printed the migration parameters and plotted a distribution diagram of the indices versus the counting layers. A detailed description of calculations has been published previously. ${ }^{17}$ Statistical analysis was carried out using paired, unpaired and one-sided t-test, Welsh test, analysis of variance (ANOVA) and linear correlations. Results are presented as mean \pm SEM and are said to be significant at $P<0.05$ if not specified otherwise. Gaussian distributions were ascertained by Chi-squared test. For comparison between RA patients and controls the appropriate values were matched.

\section{Results}

PMN migration parameters TMI and DC in RA patients compared with healthy controls are shown in Table 1 . In the control group fMLP did not influence the absolute number of migrant PMNs (unstimulated: $1238 \pm 58 \mathrm{PMN} / \mathrm{mm}^{2}$; stimulated: $1272 \pm 62 \mathrm{PMN} /$ $\mathrm{mm}^{2}$ ) and the percentage of migrating cells (TMI). Blood PMNs, ready to migrate, penetrated significantly deeper into the filters under the influence of fMLP than in blanks. DC values increased from $16.1 \pm$ 0.6 to $18.0 \pm 0.7 \mu \mathrm{m}$ after stimulation $(P<0.01)$. NMA as a product of TMI and DC was significantly elevated from $389.0 \pm 18.9$ to $442.0 \pm 21.7(P<0.05)$ in stimulated controls mainly as a result of the increased penetration depth (Fig. 1).

Blood PMNs migratory activity of RA patients generally increased compared with healthy controls.

Table 1. Total migration index (TMI) and distribution characteristic (DC) of blood PMNs in 68 migration tests as described in Patients and Methods of 15 RA patients compared with 64 healthy controls. The data represent mean values of triplicate measurements \pm SEM in unstimulated (spontaneous migration) or fMLP-stimulated samples of RA patients and healthy controls

\begin{tabular}{|c|c|c|c|c|}
\hline & & TMl & & DC \\
\hline $\begin{array}{l}\text { Controls } \\
(n=64)\end{array}$ & $\begin{array}{l}\text { Unstimulated } \\
\text { Stimulated }\end{array}$ & $\left.\begin{array}{l}24.0 \pm 0.8 \\
24.6 \pm 0.9\end{array}\right] * *$ & & $* *\left[\begin{array}{l}16.1 \pm 0.6 \\
18.0 \pm 0.7\end{array}\right]$ \\
\hline $\begin{array}{l}\text { RA patients } \\
(\boldsymbol{n}=68)\end{array}$ & $\begin{array}{l}\text { Unstimulated } \\
\text { Stimulated }\end{array}$ & $* *\left[\begin{array}{l}28.6 \pm 0.9 \\
26.1 \pm 0.9\end{array}\right.$ & $* *$ & $\begin{array}{l}22.6 \pm 1.1 \\
22.9 \pm 0.8\end{array}$ \\
\hline
\end{tabular}

${ }^{* *} P<0.01$. 


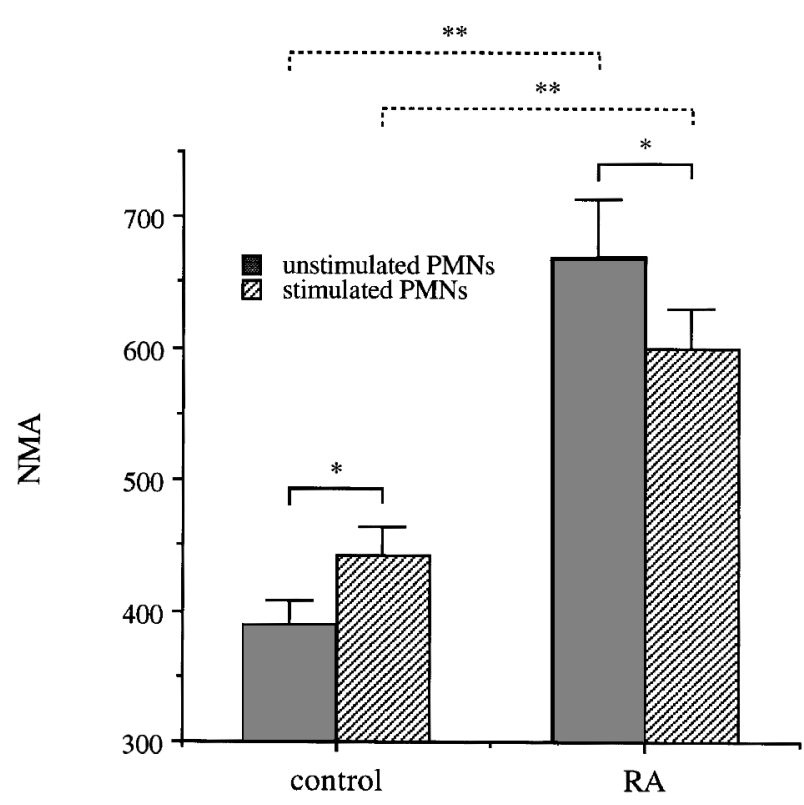

FIG. 1. Neutrophil migratory activity (NMA) of blood PMNs in 68 migration tests as described in Patients and Methods of 15 RA patients compared with 64 healthy controls. The data represent mean values of triplicate measurements \pm SEM in unstimulated (spontaneous migration) or fMLP-stimulated (directed migration) samples of RA patients and healthy controls. ${ }^{*} P<0.05,{ }^{*} P<0.01$.

Unstimulated and stimulated PMN migratory indices were significantly higher than in healthy controls except the fMLP stimulated DC, which remained unchanged (Table 1, Fig. 1). In blood samples of RA patients fMLP reduced markedly the PMN responsiveness. fMLP-stimulated values of $\mathrm{PMN} / \mathrm{mm} 2$ (unstimulated: $1954 \pm 87 \mathrm{PMN} / \mathrm{mm}^{2}$; stimulated: $1799 \pm 93$ $\mathrm{PMN} / \mathrm{mm}^{2} ; P<0.01$ ), TMI (unstimulated: $28.6 \pm 0.9 \%$ stimulated: $26.1 \pm 0.9 \%, P<0.01$ ) and NMA (unstimulated: $669.0 \pm 45.0$; stimulated: $600.0 \pm 30.0 ; P<$ 0.05 ) were significantly lower than those after spontaneous migration. Only the DC (unstimulated: $22.6 \pm$ $1.1 \mu \mathrm{m}$; stimulated: $22.9 \pm 0.1 \mu \mathrm{m})$ showed no differences between stimulated and unstimulated conditions.

A five-step grading to determine disease activity (DAC) was used (five disease stages $0=$ quiescent to $4=$ maximum activity). The number of patients in each one of the categories 0 to 4 was: DAC 0: 9 patients; DAC 1: 14 patients; DAC 2: 18 patients; DAC 3: 15 patients; DAC 4: 12 patients. Correlations between disease activity and conventional or experimental RA parameters are shown in Table 2. Disease activity calculated by ANOVA of stimulated and unstimulated values of TMI, DC and NMA did not significantly influence PMN reactivity. In contrast, the number of circulating PMNs increased with disease activity, an effect transferred to the parameter $\mathrm{PMN} / \mathrm{mm}^{2}$ essentially influenced by the $\mathrm{PMN}$ blood counts.
Table 2. RA disease activity scoring versus conventional ${ }^{\mathrm{a}}$ and experimental ${ }^{b}$ RA parameters. Regression coefficient values between disease activity and parameters are shown if significant

\begin{tabular}{|c|c|c|}
\hline \multirow[t]{2}{*}{ Parameter } & \multicolumn{2}{|c|}{ Disease activity score } \\
\hline & $\begin{array}{l}\text { regression } \\
\text { coefficients }\end{array}$ & $P$-values \\
\hline \multicolumn{3}{|l|}{ Conventional } \\
\hline ESR & 0.5573 & $<0.001$ \\
\hline CRP & 0.3831 & $<0.01$ \\
\hline $\mathrm{PMN} / \mu \mathrm{l}$ & 0.443 & $<0.001$ \\
\hline $\mathrm{HGB}$ & -0.2424 & $<0.05$ \\
\hline PLT & 0.3704 & $<0.01$ \\
\hline RF & NS & NS \\
\hline \multicolumn{3}{|c|}{$\begin{array}{l}\text { Experimental unstimulated } \\
\text { (blank control conditions) }\end{array}$} \\
\hline $\mathrm{PMN} / \mathrm{mm}^{2}$ & 0.4952 & $<0.001$ \\
\hline TMI & NS & NS \\
\hline$D C$ & NS & NS \\
\hline IVIVIA & & INS \\
\hline \multicolumn{3}{|c|}{ Experimental fMLP stimulated } \\
\hline $\mathrm{PMN} / \mathrm{mm}^{2}$ & 0.4284 & $<0.001$ \\
\hline TMl & NS & NS \\
\hline NMA & NS & NS \\
\hline
\end{tabular}

aErythrocyte sedimentation rate (ESR), C-reactive protein (CRP), blood PMNs (PMN/ $/$ l), haemoglobin (HGB), platelets (PLT) and rheumatoid factor (RF).

${ }^{b}$ Number of PMN immigrants per one square millimeter standard counting area $\left(\mathrm{PMN} / \mathrm{mm}^{2}\right)$, total migration index $(\mathrm{TMl})$, distribution characteristic (DC) and neutrophil migratory activity (NMA) as described in Patients and Methods.

$\mathrm{NS}=$ not significant, $\mathrm{fMLP}=\mathrm{N}$-formyl-methionyl-leucyl-phenylalanine .

\section{Discussion}

The long-term prognosis of RA varies from mild disability of peripheral joints to mortality rates in severe cases similar to that of patients with threevessel coronary artery disease or malignancies. ${ }^{18}$ Results of a 25-year prospective study of 209 RA patients showed a 3.7-fold increased proportional mortality ratio relative to the general population, standardized for age and sex. ${ }^{4}$ This excess mortality was associated with an increased susceptibility to bacterial infections. Suzuki et al. ${ }^{19}$ found infections as a cause of death in $24 \%$ of 81 autopsied RA patients compared with only $1.5 \%$ of 243 non-RA patients. It has been argued that this excess mortality might be in part a consequence of the disease per se. ${ }^{20}$

Circulating blood PMNs in RA are considered to be hyperactive because of their increased chemiluminescence response compared with those of healthy subjects. ${ }^{21}$ Activated PMNs emigrate from the bloodstream and migrate into articular joints where the cells are occupied with phagocytosis and the release of soluble inflammatory factors. ${ }^{22}$ Increase of PMN locomotion speed and directed migration are an essential part of the non-specific endogenous defence 
system. Experiments on infection-prone children showed defects of the PMN chemotactic response which correlated to the severity of their infections. ${ }^{23}$ Reviewing the literature we found contradictory reports of depressed, ${ }^{7-11}$ normal $^{12-14}$ or increased ${ }^{15}$ chemotax is of peripheral blood PMNs in RA patients. Differences in the technique and the isolation procedure, which might be detrimental to the function of blood PMNs as shown by Rice and Bignold ${ }^{16}$ may help to explain the controversy.

To avoid this kind of influence on PMNs, we established an experimental design that conserves the dynamic activation of PMNs. The use of diluted fresh whole blood instead of purified leukocyte fractions offers not only fast and inexpensive processing but, even more important, preserves the cells in or near their genuine priming state since they are not affected by isolation procedures.

Our results confirm the findings of Turner et al. ${ }^{10}$ This group has demonstrated a significant defect in the ability of RA PMNs to orientate in the direction of a bacterial chemoattractant gradient. Additionally Goddard et al. ${ }^{8}$ found a significant reduced chemotactic index, which was calculated by the difference of the mean value for random migration and the mean value for directed migration with the results expressed in a percentage of the control value. In contrast to this group, we were able to demonstrate a difference in the random motility of RA PMNs compared with controls.

In our results PMNs of RA patients showed a higher migratory activity with a concomitant inhibition of their reactivity towards fMLP. However, the lack of fMLP to stimulate PMNs of RA patients like PMNs of healthy controls indicates that in these patients the readiness of blood PMNs to migrate is at least partially inhibited. Itala and coworkers ${ }^{24}$ also showed reactions of PMNs of healthy controls to be stimulated by fMLP. They too found significant impairment in AMLP induced $\mathrm{PMN}$ migration in patients with chronic lymphocytic leukaemia and a history of infections. Therefore, a migration assay using AMLP as chemoattractant seems to be a suitable model to study susceptibilities to infectious diseases.

At present it is not known whether these functional anomalies of PMNs in RA patients are secondary to the disease without further pathogenetic implications or whether these anomalies are a possible pathophysiologic explanation for the increased rate of septic complications in these patients. In RA patients PMNs are directed to the inflammatory site by chemokines such as interleukin-8 and transforming grow th factor $\beta .^{25}$ These factors are detectable in synovium and blood serum of RA patients but not in healthy controls and may be partly responsible for the enhanced migratory activity of PMNs in our experiments in RA patients. Tumour necrosis factor alpha (TNF $\alpha$ ), persistently found in blood serum of RA patients, ${ }^{26}$ was able to decrease $\mathrm{AMLP}$ induced PMN chemotax is in an in vitro migration assay. ${ }^{27}$ In another study fMLP reduced PMN migration in early stages of patients with thermal injury ${ }^{28}$ where high levels of proinflammatory cytokines such as TNF $\alpha$ are produced. All these data implicate a pivotal role of TNF $\alpha$ in the decreased migratory response of AMLP stimulated PMNs observed in RA patients. Inhibitory factors different from TNF $\alpha$ may also contribute to the impaired fMLP induced PMN migration in RA. A chemokinetic inhibitory factor is expressed by B cells in chronic lymphatic leukaemia patients with a higher propensity to infections. ${ }^{29} \mathrm{~B}$ cells are activated in RA and FMLP may induce the production of such a chemokinetic inhibitory factor in our whole blood membrane filter assay explaining the reduced readiness of blood PMNs to migrate.

Recruitment of PMNs to the inflammatory site in $\mathrm{RA}$ is thought to be driven by proinflammatory cytokines mainly secreted from $\mathrm{T}$ cell activated macrophages. ${ }^{25}$ On the other hand, the capability of fMLP, as a bacteria derived peptide, to enhance PMN migratory activity seems to be a good model for an acute bacterial infection in vivo. Impaired response to $\mathrm{AMLP}$ stimulated PMNs demonstrated in our experiments may highlight what is happening to PMNs in a chronically activated immune system after challenge to bacterial infection. Further studies are encouraged to uncover mediators of inflammation which regulate the state of activation of PMNs and contribute to the diminished migration of PMNs in RA.

In conclusion, we have found an abnormal directed migration of PMNs to the bacterial chemoattractant fMLP in RA patients in vitro. Our results may help to elucidate the underlying mechanism which lead to the clinically observed increased rate of septic complications in RA.

\section{References}

1. Uddin J, Kraus AS, Kelly HG. Survivorship and death in rheumatoid arthritis. Arthritis Rheum 1970; 13: 125-130.

2. Baum J. Infection in rheumatoid arthritis. Arthritis Rheum 1971; 14: $135-137$.

3. Koota K, Isomäki $\mathrm{H}$, Mutru $\mathrm{O}$. Death rate and cause of death in rheumatoid arthritis patients during a peroid of five years. Scand J Rheumatol 1977; 6: 241-244.

4. Vandenbroucke JP, Hazevoet HM, Cats A. Survival and cause of death in rheumatoid arthritis: a 25-year prospective follow-up. J Rheumatol 1984; 11: $158-161$.

5. Mitchell DM, Spitz PW, Young DY, Bloch DA, McShane DJ, Fries JF Survival, prognosis, and causes of death in rheumatoid arthritis. Arthritis Rheum 1986; 29: 706-714.

6. Pincus T. Rheumatoid arthritis: a medical emergency? Scand J Rheumatol 1994; 23 (Suppl 100): 21-30.

7. Mowat AG, Baum J. Chemotaxis of polymorphonuclear leukocytes from patients with rheumatoid arthritis. J Clin Invest 1971; 50: 2541-2549.

8. Goddard DH, Kirk AP, Kirwan JR, Johnson GD, Holborow EJ. Impaired polymorphonulear leucocyte chemotax is in rheumatoid arthritis. Ann Rheum Dis 1984; 43: 151-156.

9. Elmgren J, Hansen TH. Subnormal sensitivity of neutrophils to complement split product C5a in rheumatoid arthritis: relation to complement catabolism and disease extent. Ann Rheum Dis 1985; 44: 514-518.

10. Turner RA, Johnson JA, Turner SR. Neutrophil responsivness to chemoattractant tripeptide in rheumatoid arthritis (42591). Proc Soc Exp Biol Med 1987; 186: 125-133. 
11. Leirisalo-Repo M, Paimela L, Koskimies S, Repo H. Function of polymorphonuclear leukocytes in early rheumatoid arthritis. Infla mmation 1993; 17: 427-442.

12. Howe GB, Fordham JN, Brown KA, Currey HLF Polymorphonuclear cell function in rheumatoid arthritis and Felty's syndrome. Ann Rheum Dis 1981; 40: 370-375.

13. King SL, Parker J, Cooper R, Sturrock R, Gemmel CG. Polymorphonuclear leukocyte function in rheumatoid arthritis. Br J Rheumatol 1986; 25: $26-33$.

14. Brown KA. The polymorphonuclear cell in rheumatoid arthritis. $B r J$ Rheumatol 1988; 27: 150-155.

15. Espersen GT, Ernst E, Vestergaard M, Pedersen JO, Grunnet N. Changes in PMN leukocyte migration activity and complement C3d levels in RA patients with high disease activity during steroid treatment. Scand J Rheumatol 1989; 18: 51-56.

16. Rice JE, Bignold LP. Chemotax is of polymorphonuclear leukocytes in whole blood in the 'sparse-pore' polycarbonate (Nuclepore) membrane/ Boyden chamber assay. J Immunol Methods 1992; 149: 121-125.

17. Egger G, Klemt C, Spendel S, Kaulfersch W, Kenzian H Migratory activity of blood polymorphnuclear leukocytes during juvenile rheumatoid arthritis, demonstrated with a new whole-blood membrane filter assay. Inflammation 1994; 18: 427-441.

18. Pincus T, Callahan LF What is the natural history of rheumatoid arthritis? Rheum Dis Clin North Am 1993; 19: 123-151.

19. Suzuki A, Ohosone Y, Obano M et al. Cause of death in 81 autopsied patients with rheumatoid arthritis. J Rheumatol 1994; 21: 33-36

20. Pincus T, Callahan LF. Quantitative measures to assess, monitor and predict morbidity and mortality in rheumatoid arthritis. Baillières Clin Rheumatol 1992; 6: 161-191.

21. Wandal JH. Leucocyte function in patients with rheumatoid arthritis: quantitative in vivo leucocyte mobilization and in vitro functions of blood and exudate leucocytes. Ann Rheum Dis 1985; 44: 694-700.
22. Weissmann G. Release of mediators of inflammation from stimulated neutrophils. N Engl J Med 1980; 303: 27-34.

23. Bondestam M, Hakansson L, Foucard T, Venge P. Defects in polymorphonuclear neutrophil function and susceptibility to infection in children. Scand J Clin Lab Invest 1986; 46: 685-694.

24. Itala M, Vainio O, Remes K. Functional abnormalities in granulocytes predict susceptibility to bacterial infections in chronic lymphocytic leukaemia. Eur J Haematol 1996; 57: 46-53.

25. Feldmann M, Brennan FM, Maini RN. Role of cytokines in rheumatoid arthritis. Annu Rev Immunol 1996; 14: 397-440.

26. Saxne T, Pallatino MA Jr, Heinegard D, Talal N, Wollheim FA. Detection of tumor necrosis factor a but not tumor necrosis factor $B$ in rheumatoid arthritis synovial fluid and serum. Arthritis Rheum 1988; 31 : 1041-1045.

27. Vollmer KL, Alberts JS, Carper HT, Mandell GL. Tumor necrosis factoralpha decreases neutrophil chemotax is to N-formyl-1-methionyl-1-leucyl1-phenylalanine: analysis of single cell movement. J Leukoc Biol 1992; 52: 630-636.

28. Vindenes HA, Bjerknes R. Impaired actin polymerization and depolymerization in neutrophils from patients with thermal injury. Burns 1997; 23: $131-136$.

29. Siegbahn A, Simonsson B, Venge P. The chemokinetic inhibitory factor (CIF) in serum of CLL patients: correlation with infection propensity and disease activity. Scand J Haematol 1985; 35: 80-87.

ACKNOWLEDGMENT: The authors w ish to thank U. Sprincnik for her help in preparing the manuscript.

\section{Received 25 August 1997;} accepted in revised form 10 October 1997 


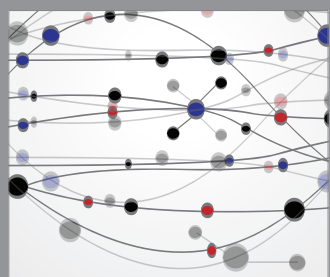

The Scientific World Journal
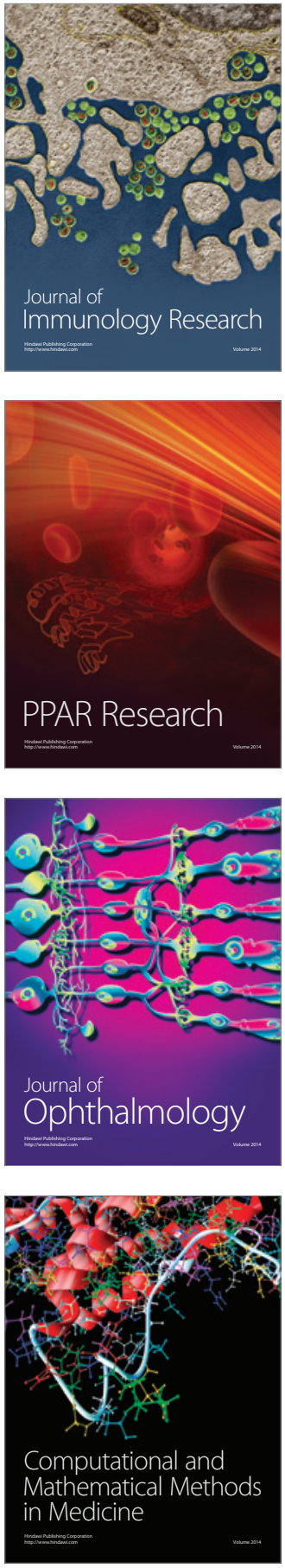

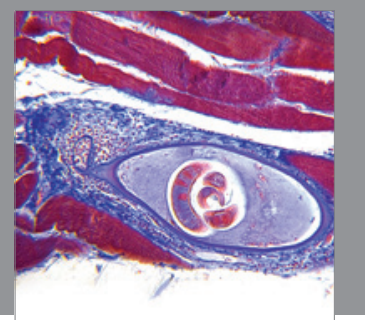

Gastroenterology

Research and Practice
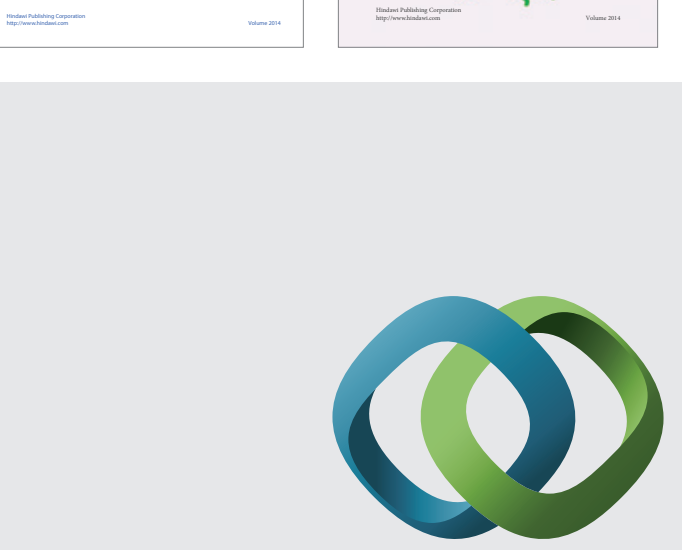

\section{Hindawi}

Submit your manuscripts at

http://www.hindawi.com
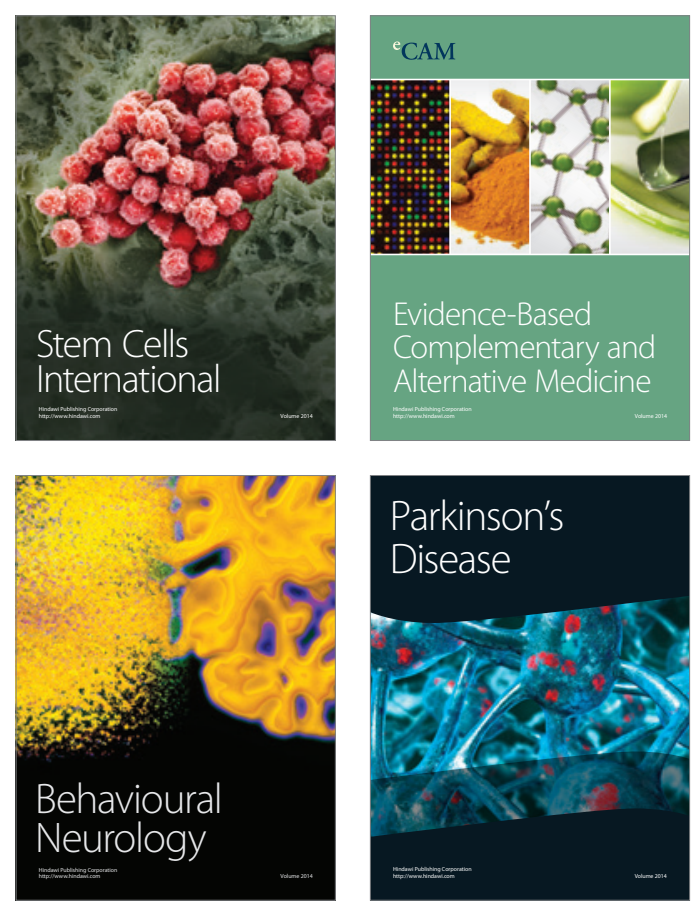

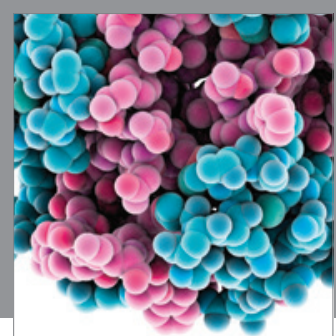

Journal of
Diabetes Research

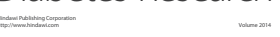

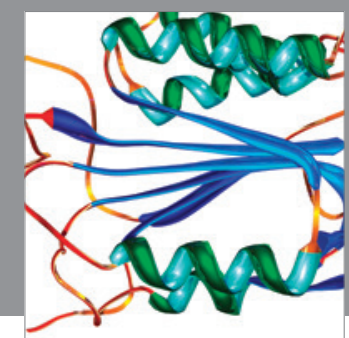

Disease Markers
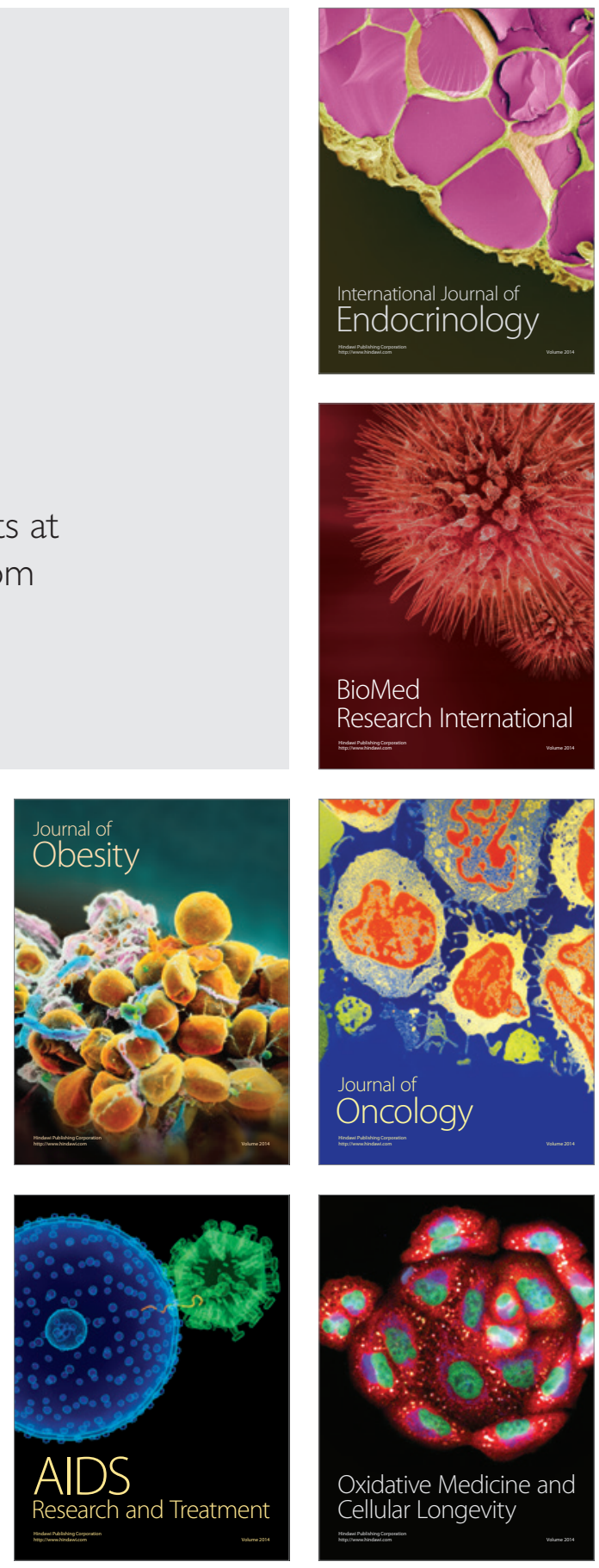